Network Working Group

Request for Comments: 5591

Category: Standards Track
D. Harrington

Huawei Technologies (USA)

W. Hardaker

Cobham Analytic Solutions

June 2009

\title{
Transport Security Model for the \\ Simple Network Management Protocol (SNMP)
}

Status of This Memo

This document specifies an Internet standards track protocol for the Internet community, and requests discussion and suggestions for improvements. Please refer to the current edition of the "Internet Official Protocol Standards" (STD 1) for the standardization state and status of this protocol. Distribution of this memo is unlimited.

Copyright Notice

Copyright (c) 2009 IETF Trust and the persons identified as the document authors. All rights reserved.

This document is subject to BCP 78 and the IETF Trust's Legal Provisions Relating to IETF Documents in effect on the date of publication of this document (http://trustee.ietf.org/license-info). Please review these documents carefully, as they describe your rights and restrictions with respect to this document.

This document may contain material from IETF Documents or IETF Contributions published or made publicly available before November 10, 2008. The person(s) controlling the copyright in some of this material may not have granted the IETF Trust the right to allow modifications of such material outside the IETF Standards Process. Without obtaining an adequate license from the person(s) controlling the copyright in such materials, this document may not be modified outside the IETF standards Process, and derivative works of it may not be created outside the IETF Standards Process, except to format it for publication as an RFC or to translate it into languages other than English. 


\section{Abstract}

This memo describes a Transport Security Model for the Simple Network Management Protocol (SNMP).

This memo also defines a portion of the Management Information Base (MIB) for monitoring and managing the Transport security Model for

SNMP.

Table of Contents

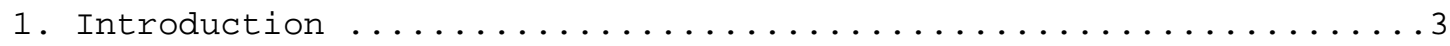

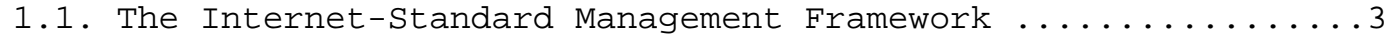

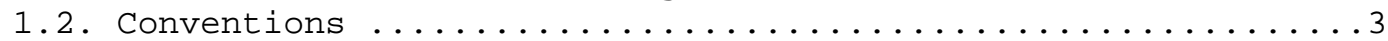

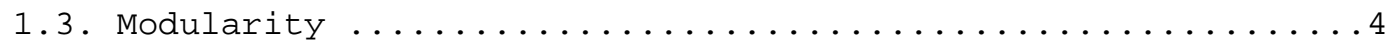

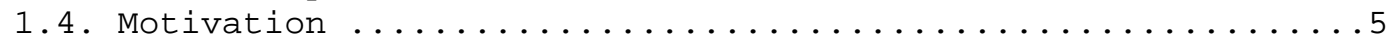

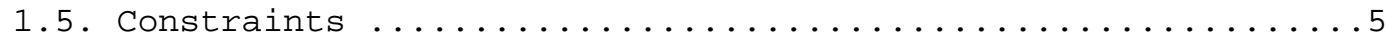

2. How the Transport Security Model Fits in the Architecture ....6

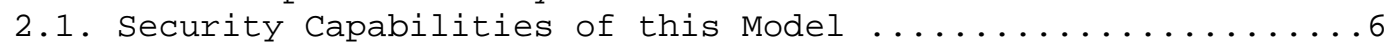

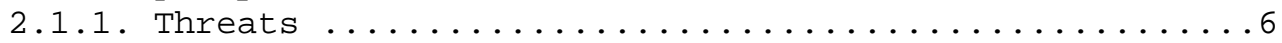

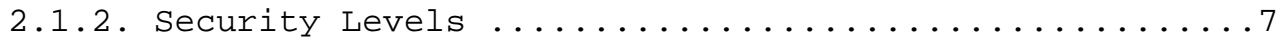

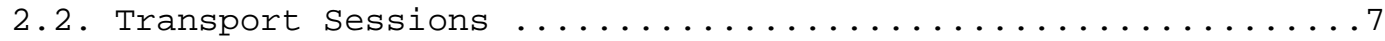

2.3. Coexistence ..........................

2.3.1. Coexistence with Message Processing Models .......7

2.3.2. Coexistence with Other Security Models ..........8

2.3.3. Coexistence with Transport Models .............

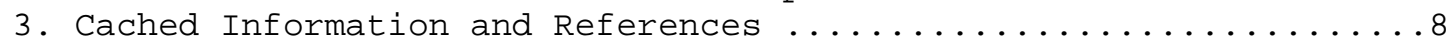

3.1. Transport Security Model Cached Information ...........99

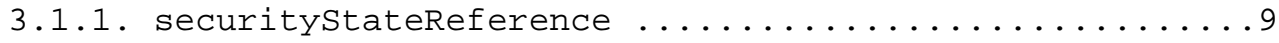

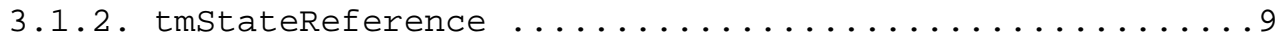

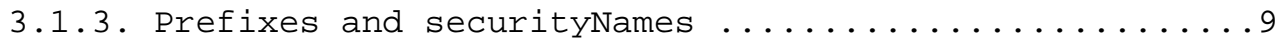

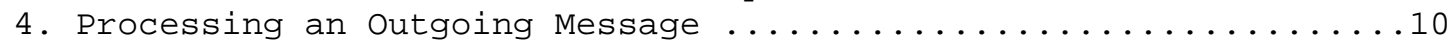

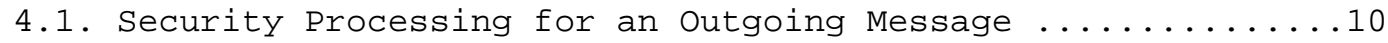

4.2. Elements of Procedure for Outgoing Messages ...........11

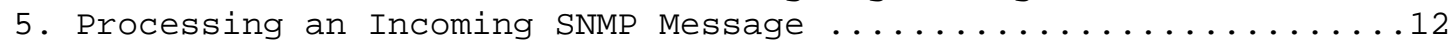

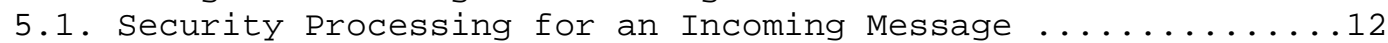

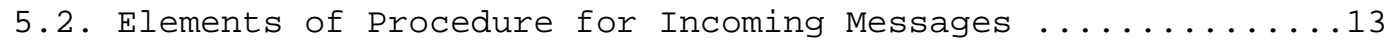

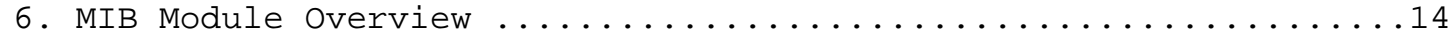

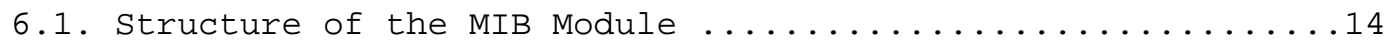

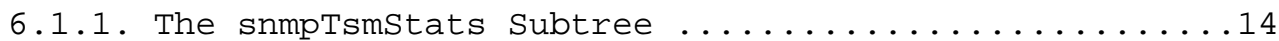

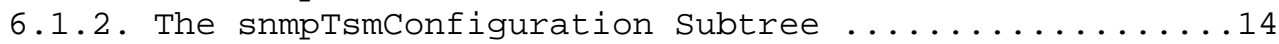

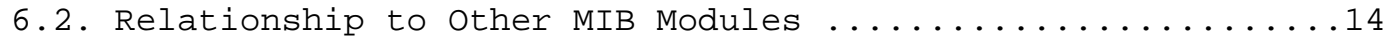

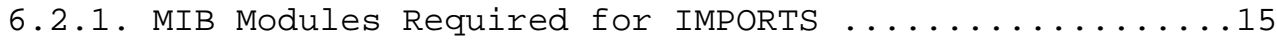

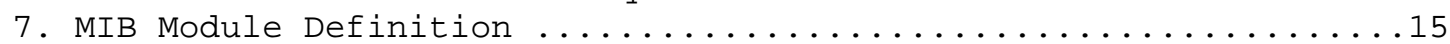

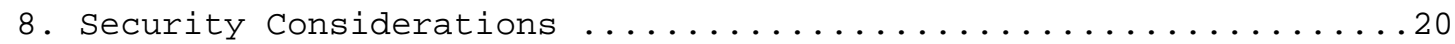

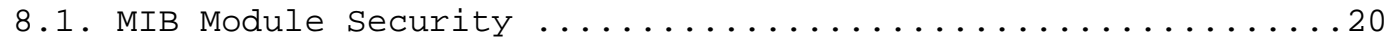

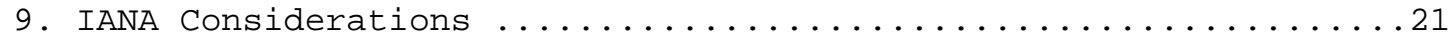

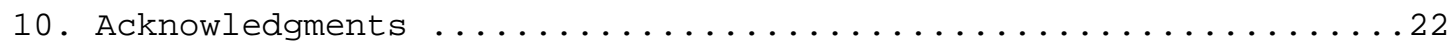




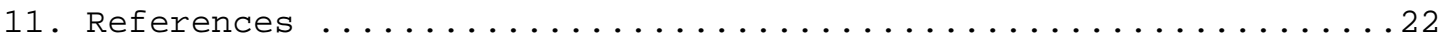

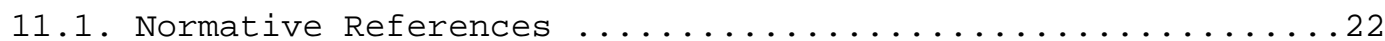

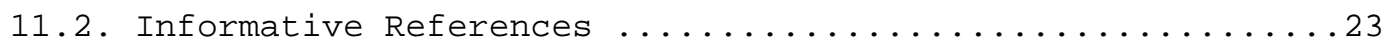

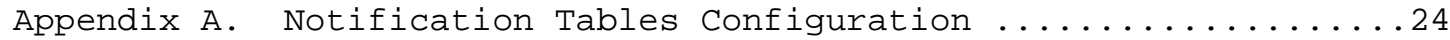

A.1. Transport Security Model Processing for Notifications ....25

Appendix B. Processing Differences between USM and Secure

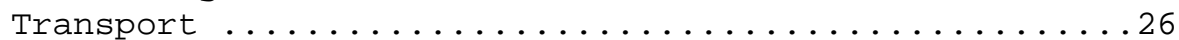

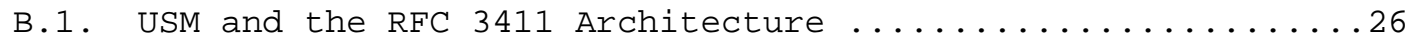

B.2. Transport Subsystem and the RFC 3411 Architecture ......27

1. Introduction

This memo describes a Transport Security Model for the Simple Network Management Protocol for use with secure Transport Models in the Transport Subsystem [RFC5590].

This memo also defines a portion of the Management Information Base (MIB) for monitoring and managing the Transport security Model for SNMP.

It is important to understand the SNMP architecture and the terminology of the architecture to understand where the Transport Security Model described in this memo fits into the architecture and interacts with other subsystems and models within the architecture. It is expected that readers will have also read and understood [RFC3411], [RFC3412], [RFC3413], and [RFC3418].

\subsection{The Internet-Standard Management Framework}

For a detailed overview of the documents that describe the current Internet-Standard Management Framework, please refer to section 7 of RFC 3410 [RFC3410].

Managed objects are accessed via a virtual information store, termed the Management Information Base or MIB. MIB objects are generally accessed through the Simple Network Management Protocol (SNMP). Objects in the MIB are defined using the mechanisms defined in the Structure of Management Information (SMI). This memo specifies a MIB module that is compliant to the SMIV2, which is described in STD 58, RFC 2578 [RFC2578], STD 58, RFC 2579 [RFC2579] and STD 58, RFC 2580 [RFC2580].

\subsection{Conventions}

The key words "MUST", "MUST NOT", "REQUIRED", "SHALL", "SHALL NOT", "SHOULD", "SHOULD NOT", "RECOMMENDED", "MAY", and "OPTIONAL" in this document are to be interpreted as described in [RFC2119]. 
Lowercase versions of the keywords should be read as in normal English. They will usually, but not always, be used in a context that relates to compatibility with the RFC 3411 architecture or the subsystem defined here but that might have no impact on on-the-wire compatibility. These terms are used as guidance for designers of proposed IETF models to make the designs compatible with RFC 3411 subsystems and Abstract Service Interfaces (ASIs). Implementers are free to implement differently. Some usages of these lowercase terms are simply normal English usage.

For consistency with SNMP-related specifications, this document favors terminology as defined in STD 62, rather than favoring terminology that is consistent with non-SNMP specifications that use different variations of the same terminology. This is consistent with the IESG decision to not require the SNMPv3 terminology be modified to match the usage of other non-SNMP specifications when SNMPv3 was advanced to Full standard.

Authentication in this document typically refers to the English meaning of "serving to prove the authenticity of" the message, not data source authentication or peer identity authentication.

The terms "manager" and "agent" are not used in this document because, in the RFC 3411 architecture, all SNMP entities have the capability of acting as manager, agent, or both depending on the SNMP applications included in the engine. Where distinction is needed, the application names of command generator, command responder, notification originator, notification receiver, and proxy forwarder are used. See "Simple Network Management Protocol (SNMP) Applications" [RFC3413] for further information.

While security protocols frequently refer to a user, the terminology used in [RFC3411] and in this memo is "principal". A principal is the "who" on whose behalf services are provided or processing takes place. A principal can be, among other things, an individual acting in a particular role, a set of individuals each acting in a particular role, an application or a set of applications, or a combination of these within an administrative domain.

\subsection{Modularity}

The reader is expected to have read and understood the description of the SNMP architecture, as defined in [RFC3411], and the architecture extension specified in "Transport Subsystem for the Simple Network Management Protocol (SNMP)" [RFC5590], which enables the use of external "lower-layer transport" protocols to provide message 
security. Transport Models are tied into the SNMP architecture through the Transport Subsystem. The Transport Security Model is designed to work with such lower-layer, secure Transport Models.

In keeping with the RFC 3411 design decisions to use self-contained documents, this memo includes the elements of procedure plus associated MIB objects that are needed for processing the Transport Security Model for SNMP. These MIB objects SHOULD NOT be referenced in other documents. This allows the Transport Security Model to be designed and documented as independent and self-contained, having no direct impact on other modules. It also allows this module to be upgraded and supplemented as the need arises, and to move along the standards track on different time-lines from other modules.

This modularity of specification is not meant to be interpreted as imposing any specific requirements on implementation.

\subsection{Motivation}

This memo describes a Security Model to make use of Transport Models that use lower-layer, secure transports and existing and commonly deployed security infrastructures. This security Model is designed to meet the security and operational needs of network administrators, maximize usability in operational environments to achieve high deployment success, and at the same time minimize implementation and deployment costs to minimize the time until deployment is possible.

\subsection{Constraints}

The design of this SNMP Security Model is also influenced by the following constraints:

1. In times of network stress, the security protocol and its underlying security mechanisms SHOULD NOT depend solely upon the ready availability of other network services (e.g., Network Time Protocol (NTP) or Authentication, Authorization, and Accounting (AAA) protocols).

2. When the network is not under stress, the Security Model and its underlying security mechanisms MAY depend upon the ready availability of other network services.

3. It might not be possible for the Security Model to determine when the network is under stress.

4. A Security Model SHOULD NOT require changes to the SNMP architecture. 
5. A Security Model SHOULD NOT require changes to the underlying security protocol.

2. How the Transport Security Model Fits in the Architecture

The Transport Security Model is designed to fit into the RFC 3411 architecture as a security Model in the security subsystem and to utilize the services of a secure Transport Model.

For incoming messages, a secure Transport Model will pass a tmstateReference cache, described in [RFC5590]. To maintain RFC 3411 modularity, the Transport Model will not know which securityModel will process the incoming message; the Message Processing Model will determine this. If the Transport Security Model is used with a nonsecure Transport Model, then the cache will not exist or will not be populated with security parameters, which will cause the Transport Security Model to return an error (see section 5.2).

The Transport Security Model will create the securityName and securitylevel to be passed to applications, and will verify that the tmTransportSecurityLevel reported by the Transport Model is at least as strong as the securitylevel requested by the Message Processing Model.

For outgoing messages, the Transport Security Model will create a tmStateReference cache (or use an existing one), and will pass the tmstateReference to the specified Transport Model.

\subsection{Security Capabilities of this Model}

\subsubsection{Threats}

The Transport Security Model is compatible with the RFC 3411 architecture and provides protection against the threats identified by the RFC 3411 architecture. However, the Transport Security Model does not provide security mechanisms such as authentication and encryption itself. Which threats are addressed and how they are mitigated depends on the Transport Model used. To avoid creating potential security vulnerabilities, operators should configure their system so this Security Model is always used with a Transport Model that provides appropriate security, where "appropriate" for a particular deployment is an administrative decision. 


\subsubsection{Security Levels}

The RFC 3411 architecture recognizes three levels of security:

- without authentication and without privacy (noAuthNoPriv)

- with authentication but without privacy (authNoPriv)

- with authentication and with privacy (authPriv)

The model-independent securitylevel parameter is used to request specific levels of security for outgoing messages and to assert that specific levels of security were applied during the transport and processing of incoming messages.

The transport-layer algorithms used to provide security should not be exposed to the Transport Security Model, as the Transport Security Model has no mechanisms by which it can test whether an assertion made by a Transport Model is accurate.

The Transport Security Model trusts that the underlying secure transport connection has been properly configured to support security characteristics at least as strong as reported in tmTransportsecurityLevel.

\subsection{Transport Sessions}

The Transport Security Model does not work with transport sessions directly. Instead the transport-related state is associated with a unique combination of transportDomain, transportAddress, securityName, and securitylevel, and is referenced via the tmstateReference parameter. How and if this is mapped to a particular transport or channel is the responsibility of the Transport Subsystem.

\subsection{Coexistence}

In the RFC 3411 architecture, a Message Processing Model determines which security Model sHALL be called. As of this writing, IANA has registered four Message Processing Models (SNMPv1, SNMPv2c, SNMPv2u/ SNMPv2*, and SNMPv3) and three other Security Models (SNMPv1, SNMPv2c, and the User-based Security Model).

\subsubsection{Coexistence with Message Processing Models}

The SNMPV1 and SNMPV2C message processing described in BCP 74 [RFC3584] always selects the SNMPv1(1) and SNMPv2c(2) Security Models. Since there is no mechanism defined in RFC 3584 to select an 
alternative Security Model, SNMPv1 and SNMPv2c messages cannot use the Transport Security Model. Messages might still be able to be conveyed over a secure transport protocol, but the Transport security Model will not be invoked.

The SNMPv2u/SNMPv2* Message Processing Model is an historic artifact for which there is no existing IETF specification.

The SNMPv3 message processing defined in [RFC3412] extracts the securityModel from the msgSecurityModel field of an incoming SNMPv3Message. When this value is transportsecurityModel(4), security processing is directed to the Transport Security Model. For an outgoing message to be secured using the Transport Security Model, the application MUST specify a securityModel parameter value of transportsecurityModel(4) in the sendPdu Abstract Service Interface $(\mathrm{ASI})$.

\subsubsection{Coexistence with Other Security Models}

The Transport Security Model uses its own MIB module for processing to maintain independence from other security Models. This allows the Transport Security Model to coexist with other Security Models, such as the User-based Security Model (USM) [RFC3414].

\subsubsection{Coexistence with Transport Models}

The Transport Security Model (TSM) MAY work with multiple Transport Models, but the RFC 3411 Abstract Service Interfaces (ASIs) do not carry a value for the Transport Model. The MIB module defined in this memo allows an administrator to configure whether or not TSM prepends a Transport Model prefix to the securityName. This will allow SNMP applications to consider Transport Model as a factor when making decisions, such as access control, notification generation, and proxy forwarding.

To have SNMP properly utilize the security services coordinated by the Transport Security Model, this Security Model MUST only be used with Transport Models that know how to process a tmStateReference, such as the Secure Shell Transport Model [RFC5592].

3. Cached Information and References

When performing SNMP processing, there are two levels of state information that might need to be retained: the immediate state linking a request-response pair and a potentially longer-term state relating to transport and security. "Transport subsystem for the Simple Network Management Protocol (SNMP)" [RFC5590] defines general requirements for caches and references. 
This document defines additional cache requirements related to the Transport Security Model.

\subsection{Transport Security Model Cached Information}

The Transport Security Model has specific responsibilities regarding the cached information.

\subsection{1. securitystateReference}

The Transport Security Model adds the tmStateReference received from the processIncomingMsg ASI to the securitystateReference. This tmStateReference can then be retrieved during the generateResponseMsg ASI so that it can be passed back to the Transport Model.

\subsection{2. tmStateReference}

For outgoing messages, the Transport Security Model uses parameters provided by the SNMP application to look up or create a tmstateReference.

For the Transport Security Model, the security parameters used for a response MUST be the same as those used for the corresponding request. This Security Model uses the tmStateReference stored as part of the securitystateReference when appropriate. For responses and reports, this security Model sets the tmSamesecurity flag to true in the tmStateReference before passing it to a Transport Model.

For incoming messages, the Transport Security Model uses parameters provided in the tmStateReference cache to establish a securityName, and to verify adequate security levels.

\subsubsection{Prefixes and securityNames}

The SNMP-VIEW-BASED-ACM-MIB module [RFC3415], the SNMP-TARGET-MIB module [RFC3413], and other MIB modules contain objects to configure security parameters for use by applications such as access control, notification generation, and proxy forwarding.

Transport domains and their corresponding prefixes are coordinated via the IANA registry "SNMP Transport Domains".

If snmpTsmConfigurationUsePrefix is set to true, then all securityNames provided by, or provided to, the Transport security Model MUST include a valid transport domain prefix. 
If snmpTsmConfigurationUsePrefix is set to false, then all securityNames provided by, or provided to, the Transport security Model MUST NOT include a transport domain prefix.

The tmSecurityName in the tmStateReference stored as part of the securitystateReference does not contain a prefix.

4. Processing an Outgoing Message

An error indication might return an object Identifier (OID) and value for an incremented counter, a value for securitylevel, values for contextEngineID and contextName for the counter, and the securitystateReference, if this information is available at the point where the error is detected.

\subsection{Security Processing for an Outgoing Message}

This section describes the procedure followed by the Transport Security Model.

The parameters needed for generating a message are supplied to the Security Model by the Message Processing Model via the generateRequestMsg() or the generateResponseMsg() ASI. The Transport Subsystem architectural extension has added the transportDomain, transportAddress, and tmStateReference parameters to the original RFC 3411 ASIs.

statusInformation $=$ generateRequestMsg(

IN messageProcessingModel

IN globalData

IN maxMessagesize

IN transportDomain

IN transportAddress

IN securityModel

IN securityEngineID

IN securityName

IN securityLevel

IN scopedPDU

OUT securityParameters

OUT wholeMsg

OUT wholeMsgLength

OUT tmStateReference )
-- success or errorIndication

-- typically, SNMP version

-- message header, admin data

-- of the sending SNMP entity

-- (NEW) specified by application

-- (NEW) specified by application

-- for the outgoing message

-- authoritative SNMP entity

-- on behalf of this principal

-- Level of Security requested

-- message (plaintext) payload

-- filled in by Security Module

- complete generated message

- length of generated message

-- (NEW) transport info

statusInformation $=--$ success or errorIndication

generateResponseMsg(

IN messageProcessingModel -- typically, SNMP version 


$\begin{array}{ll}\text { IN } & \text { globalData } \\ \text { IN } & \text { maxMessagesize } \\ \text { IN } & \text { transportDomain } \\ \text { IN } & \text { transportAddress } \\ \text { IN } & \text { securityModel } \\ \text { IN } & \text { securityEngineID } \\ \text { IN } & \text { securityName } \\ \text { IN } & \text { securityLevel } \\ \text { IN } & \text { scopedPDU } \\ \text { IN } & \text { securityStateReference } \\ & \\ & \\ \text { OUT } & \text { securityParameters } \\ \text { OUT } & \text { wholeMsg } \\ \text { OUT } & \text { wholeMsgLength } \\ \text { OUT } & \text { tmStateReference } \\ & \text { ) }\end{array}$

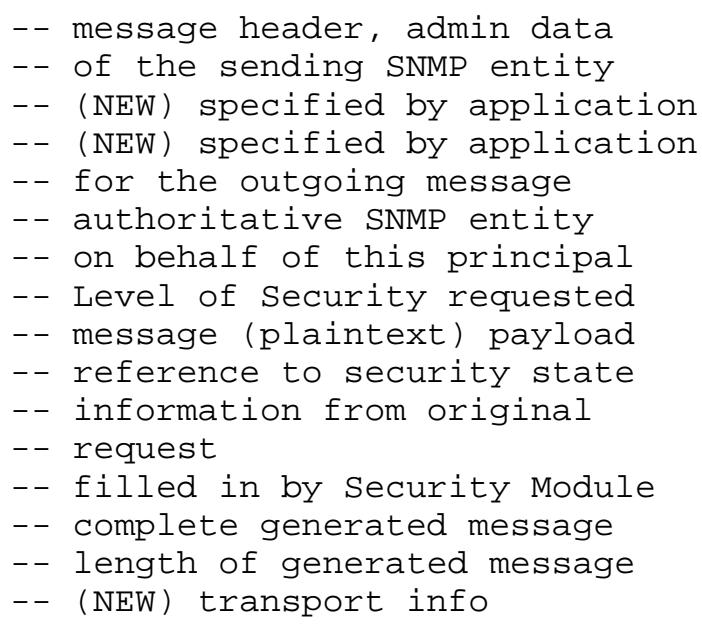

4.2. Elements of Procedure for Outgoing Messages

1. If there is a securitystateReference (Response or Report message), then this security Model uses the cached information rather than the information provided by the ASI. Extract the tmStateReference from the securitystateReference cache. Set the tmRequestedSecurityLevel to the value of the extracted tmiransportsecuritylevel. Set the tmSameSecurity parameter in the tmStateReference cache to true. The cachedsecurityData for this message can now be discarded.

2. If there is no securityStateReference (e.g., a Request-type or Notification message), then create a tmStateReference cache. Set tmTransportDomain to the value of transportDomain, tmTransportAddress to the value of transportAddress, and tmRequestedSecurityLevel to the value of securityLevel. (Implementers might optimize by pointing to saved copies of these session-specific values.) Set the transaction-specific tmamesecurity parameter to false.

If the snmpTsmConfigurationUsePrefix object is set to false, then set tmsecurityName to the value of securityName.

If the snmpTsmConfigurationUsePrefix object is set to true, then use the transportDomain to look up the corresponding prefix. (Since the securitystateReference stores the tmStateReference with the tmSecurityName for the incoming message, and since tmSecurityName never has a prefix, the prefix-stripping step only occurs when we are not using the securitystateReference). 
If the prefix lookup fails for any reason, then the snmpTsmUnknownPrefixes counter is incremented, an error indication is returned to the calling module, and message processing stops.

If the lookup succeeds, but there is no prefix in the securityName, or the prefix returned does not match the prefix in the securityName, or the length of the prefix is less than 1 or greater than 4 US-ASCII alpha-numeric characters, then the snmptsmInvalidPrefixes counter is incremented, an error indication is returned to the calling module, and message processing stops.

Strip the transport-specific prefix and trailing ' :' character (US-ASCII 0x3a) from the securityName. Set tmSecurityName to the value of securityName.

3. Set securityParameters to a zero-length OCTET STRING ('0400').

4. Combine the message parts into a wholeMsg and calculate wholeMsgLength.

5. The wholeMsg, wholeMsgLength, securityParameters, and tmStateReference are returned to the calling Message Processing Model with the statusinformation set to success.

5. Processing an Incoming SNMP Message

An error indication might return an OID and value for an incremented counter, a value for securitylevel, values for contextengineID and contextName for the counter, and the securitystateReference, if this information is available at the point where the error is detected.

\subsection{Security Processing for an Incoming Message}

This section describes the procedure followed by the Transport Security Model whenever it receives an incoming message from a Message Processing Model. The ASI from a Message Processing Model to the security subsystem for a received message is:

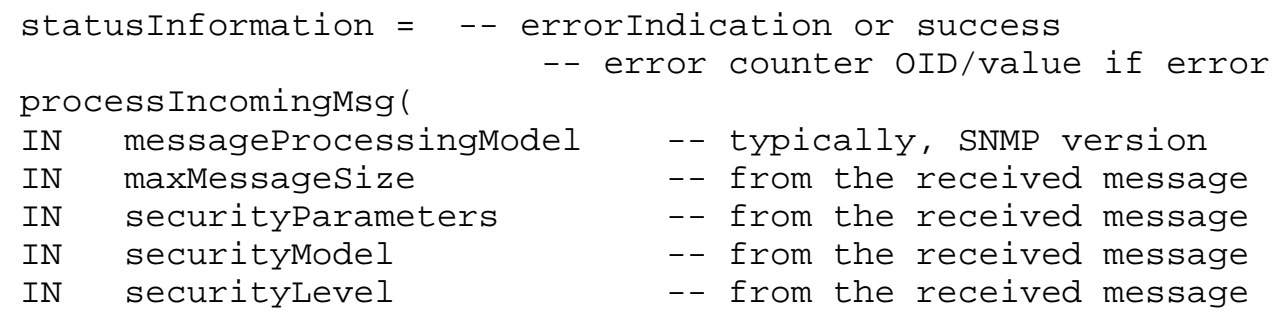



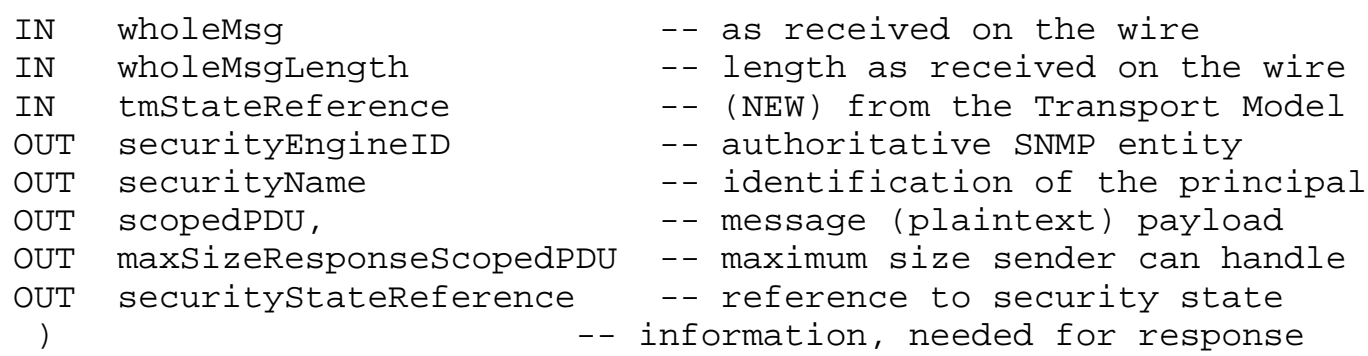

\subsection{Elements of Procedure for Incoming Messages}

1. Set the securityEngineID to the local snmpEngineID.

2. If tmStateReference does not refer to a cache containing values for tmTransportDomain, tmTransportAddress, tmSecurityName, and tmTransportSecurityLevel, then the snmpTsmInvalidCaches counter is incremented, an error indication is returned to the calling module, and Security Model processing stops for this message.

3. Copy the tmSecurityName to securityName.

If the snmptsmConfigurationUsePrefix object is set to true, then use the tmTransportDomain to look up the corresponding prefix.

If the prefix lookup fails for any reason, then the snmpTsmunknownPrefixes counter is incremented, an error indication is returned to the calling module, and message processing stops.

If the lookup succeeds but the prefix length is less than 1 or greater than 4 octets, then the snmpTsmInvalidPrefixes counter is incremented, an error indication is returned to the calling module, and message processing stops.

Set the securityName to be the concatenation of the prefix, a ' $:$ ' character (US-ASCII 0x3a), and the tmSecurityName.

4. Compare the value of tmTransportsecurityLevel in the tmStateReference cache to the value of the securityLevel parameter passed in the processIncomingMsg ASI. If securityLevel specifies privacy (Priv) and tmTransportsecurityLevel specifies no privacy (nopriv), or if securitylevel specifies authentication (auth) and tmTransportSecuritylevel specifies no authentication (noAuth) was provided by the Transport Model, then the snmpTsmInadequatesecurityLevels counter is incremented, an error indication (unsupportedSecurityLevel) together with the OID and 
value of the incremented counter is returned to the calling module, and Transport Security Model processing stops for this message.

5. The tmStateReference is cached as cachedSecurityData so that a possible response to this message will use the same security parameters. Then securitystateReference is set for subsequent references to this cached data.

6. The scopedPDU component is extracted from the wholeMsg.

7. The maxSizeResponseScopedPDU is calculated. This is the maximum size allowed for a scopedPDU for a possible Response message.

8. The statusinformation is set to success and a return is made to the calling module passing back the OUT parameters as specified in the processIncomingMsg ASI.

6. MIB Module Overview

This MIB module provides objects for use only by the Transport Security Model. It defines a configuration scalar and related error counters.

6.1. Structure of the MIB Module

Objects in this MIB module are arranged into subtrees. Each subtree is organized as a set of related objects. The overall structure and assignment of objects to their subtrees, and the intended purpose of each subtree, is shown below.

6.1.1. The snmptsmstats subtree

This subtree contains error counters specific to the Transport Security Model.

6.1.2. The snmptsmConfiguration Subtree

This subtree contains a configuration object that enables administrators to specify if they want a transport domain prefix prepended to securityNames for use by applications.

\subsection{Relationship to Other MIB Modules}

Some management objects defined in other MIB modules are applicable to an entity implementing the Transport Security Model. In particular, it is assumed that an entity implementing the Transport Security Model will implement the SNMP-FRAMEWORK-MIB [RFC3411], the 
SNMP-TARGET-MIB [RFC3413], the SNMP-VIEW-BASED-ACM-MIB [RFC3415], and the SNMPV2-MIB [RFC3418]. These are not needed to implement the SNMP-TSM-MIB .

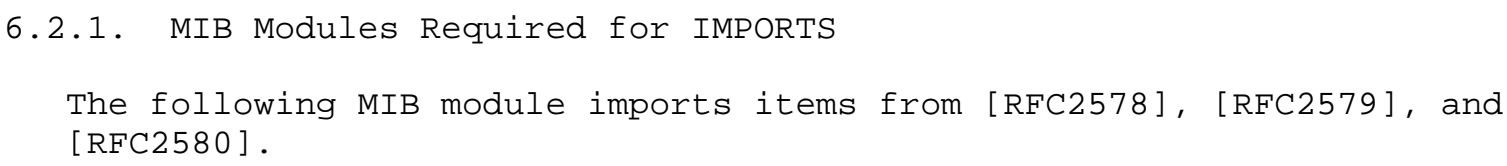




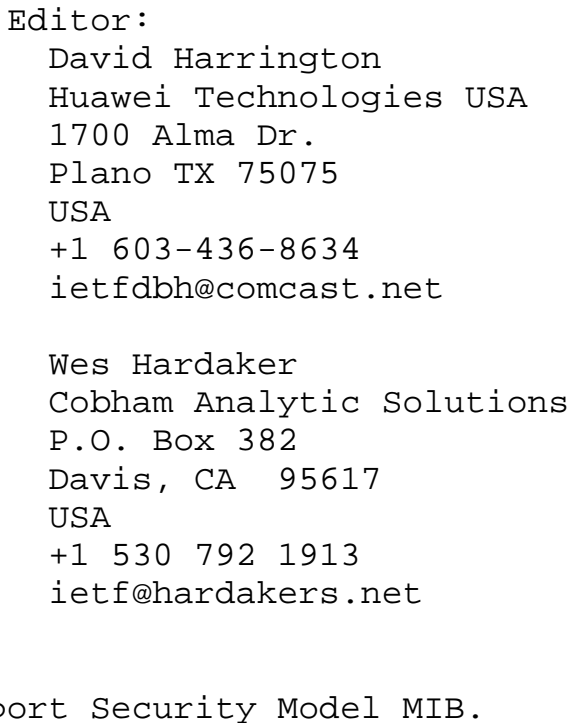

"The Transport Security Model MIB.

In keeping with the RFC 3411 design decisions to use self-contained documents, the RFC that contains the definition of this MIB module also includes the elements of procedure that are needed for processing the Transport Security Model for SNMP. These MIB objects SHOULD NOT be modified via other subsystems or models defined in other documents. This allows the Transport Security Model for SNMP to be designed and documented as independent and self-contained, having no direct impact on other modules, and this allows this module to be upgraded and supplemented as the need arises, and to move along the standards track on different time-lines from other modules.

Copyright (c) 2009 IETF Trust and the persons identified as authors of the code. All rights reserved.

Redistribution and use in source and binary forms, with or without modification, are permitted provided that the following conditions are met:

- Redistributions of source code must retain the above copyright notice, this list of conditions and the following disclaimer.

- Redistributions in binary form must reproduce the above copyright notice, this list of conditions and the following disclaimer in the documentation and/or other materials provided with the distribution. 
- Neither the name of Internet Society, IETF or IETF Trust, nor the names of specific contributors, may be used to endorse or promote products derived from this software without specific prior written permission.

THIS SOFTWARE IS PROVIDED BY THE COPYRIGHT HOLDERS AND CONTRIBUTORS 'AS IS' AND ANY EXPRESS OR IMPLIED WARRANTIES, INCLUDING, BUT NOT LIMITED TO, THE IMPLIED WARRANTIES OF MERCHANTABILITY AND FITNESS FOR A PARTICULAR PURPOSE ARE DISCLAIMED. IN NO EVENT SHALL THE COPYRIGHT OWNER OR CONTRIBUTORS BE LIABLE FOR ANY DIRECT, INDIRECT, INCIDENTAL, SPECIAL, EXEMPLARY, OR CONSEQUENTIAL DAMAGES (INCLUDING, BUT NOT LIMITED TO, PROCUREMENT OF SUBSTITUTE GOODS OR SERVICES; LOSS OF USE, DATA, OR PROFITS; OR BUSINESS INTERRUPTION) HOWEVER CAUSED AND ON ANY THEORY OF LIABILITY, WHETHER IN CONTRACT, STRICT LIABILITY, OR TORT (INCLUDING NEGLIGENCE OR OTHERWISE) ARISING IN ANY WAY OUT OF THE USE OF THIS SOFTWARE, EVEN IF ADVISED OF THE POSSIBILITY OF SUCH DAMAGE.

This version of this MIB module is part of RFC 5591; see the RFC itself for full legal notices."

REVISION "200906090000Z"

DESCRIPTION "The initial version, published in RFC 5591."

$::=\{\operatorname{mib}-2190\}$

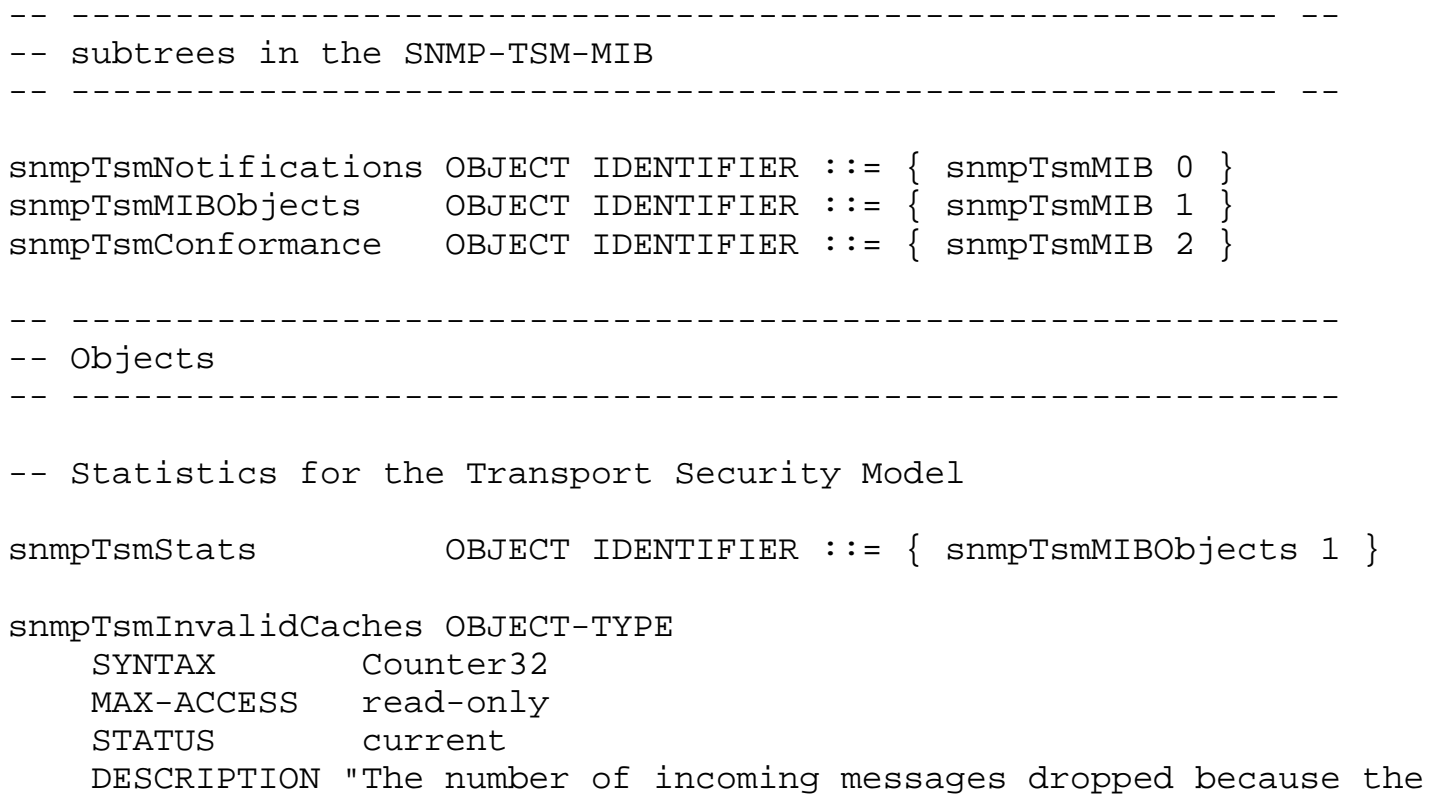




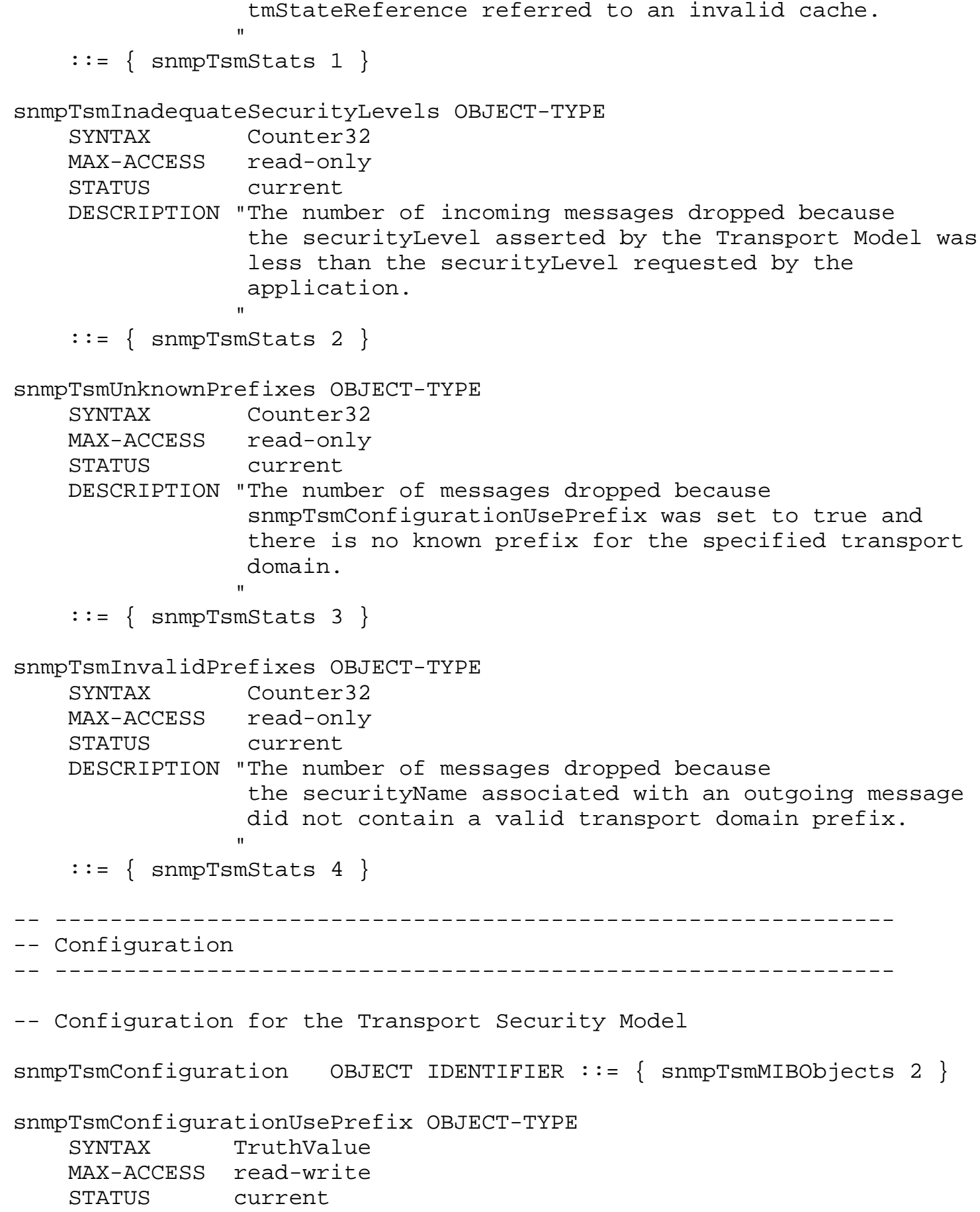




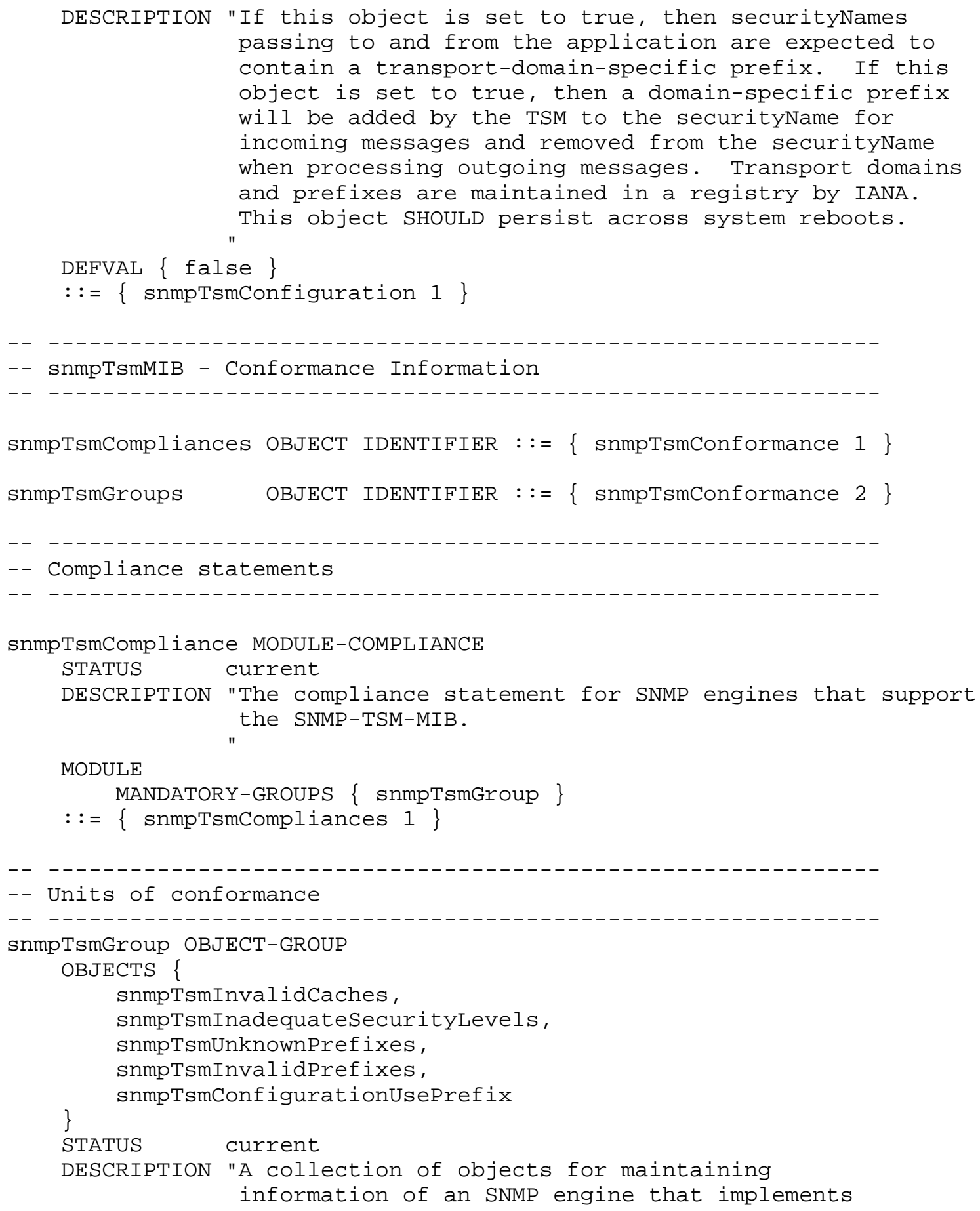




\section{"}

$::=\{$ snmpTsmGroups 2$\}$

END

8. Security Considerations

This document describes a Security Model, compatible with the RFC 3411 architecture, that permits SNMP to utilize security services provided through an SNMP Transport Model. The Transport Security Model relies on Transport Models for mutual authentication, binding of keys, confidentiality, and integrity.

The Transport Security Model relies on secure Transport Models to provide an authenticated principal identifier and an assertion of whether authentication and privacy are used during transport. This Security Model SHOULD always be used with Transport Models that provide adequate security, but "adequate security" is a configuration and/or run-time decision of the operator or management application. The security threats and how these threats are mitigated should be covered in detail in the specifications of the Transport Models and the underlying secure transports.

An authenticated principal identifier (securityName) is used in SNMP applications for purposes such as access control, notification generation, and proxy forwarding. This security Model supports multiple Transport Models. Operators might judge some transports to be more secure than others, so this Security Model can be configured to prepend a prefix to the securityName to indicate the Transport Model used to authenticate the principal. Operators can use the prefixed securityName when making application decisions about levels of access.

\subsection{MIB Module Security}

There are a number of management objects defined in this MIB module with a MAX-ACCESS clause of read-write and/or read-create. Such objects may be considered sensitive or vulnerable in some network environments. The support for SET operations in a non-secure environment without proper protection can have a negative effect on network operations. These are the tables and objects and their sensitivity/vulnerability: 
- The snmptsmConfigurationUsePrefix object could be modified, creating a denial of service or authorizing SNMP messages that would not have previously been authorized by an Access Control Model (e.g., the View-based Access Control Model (VACM)).

Some of the readable objects in this MIB module (i.e., objects with a MAX-ACCESS other than not-accessible) may be considered sensitive or vulnerable in some network environments. It is thus important to control even GET and/or NOTIFY access to these objects and possibly to even encrypt the values of these objects when sending them over the network via SNMP. These are the tables and objects and their sensitivity/vulnerability:

- All the counters in this module refer to configuration errors and do not expose sensitive information.

SNMP versions prior to SNMPv3 did not include adequate security. Even if the network itself is secure (for example by using IPsec), even then, there is no control as to who on the secure network is allowed to access and GET/SET (read/change/create/delete) the objects in this MIB module.

It is RECOMMENDED that implementers consider the security features as provided by the SNMPV3 framework (see [RFC3410], section 8), including full support for the USM and Transport Security Model cryptographic mechanisms (for authentication and privacy).

Further, deployment of SNMP versions prior to SNMPv3 is NOT RECOMMENDED. Instead, it is RECOMMENDED to deploy SNMPv3 and to enable cryptographic security. It is then a customer/operator responsibility to ensure that the SNMP entity giving access to an instance of this MIB module is properly configured to give access to the objects only to those principals (users) that have legitimate rights to indeed GET or SET (change/create/delete) them.

9. IANA Considerations

IANA has assigned:

1. An SMI number (190) with a prefix of mib-2 in the MIB module registry for the MIB module in this document.

2. A value (4) to identify the Transport Security Model, in the Security Models registry of the SNMP Number spaces registry. This results in the following table of values: 


$\begin{array}{clc}\text { Value } & \text { Description } & \text { References } \\ ---- & -------- & ------- \\ 0 & \text { reserved for 'any' } & \text { [RFC3411] } \\ 1 & \text { reserved for SNMPv1 } & \text { [RFC3411] } \\ 2 & \text { reserved for SNMPv2C } & \text { [RFC3411] } \\ 3 & \text { User-Based Security Model (USM) } & \text { [RFC3411] } \\ 4 & \text { Transport Security Model (TSM) } & \text { [RFC5591] }\end{array}$

10. Acknowledgments

The editors would like to thank Jeffrey Hutzelman for sharing his SSH insights and Dave Shield for an outstanding job wordsmithing the existing document to improve organization and clarity.

Additionally, helpful document reviews were received from Juergen Schoenwaelder.

11. References

11.1. Normative References

[RFC2119] Bradner, S., "Key words for use in RFCs to Indicate Requirement Levels", BCP 14, RFC 2119, March 1997.

[RFC2578] McCloghrie, K., Ed., Perkins, D., Ed., and J. Schoenwaelder, Ed., "Structure of Management Information Version 2 (SMIv2)", STD 58, RFC 2578, April 1999.

[RFC2579] McCloghrie, K., Ed., Perkins, D., Ed., and J. Schoenwaelder, Ed., "Textual Conventions for SMIv2", STD 58, RFC 2579, April 1999.

[RFC2580] McCloghrie, K., Perkins, D., and J. Schoenwaelder, "Conformance Statements for SMIv2", STD 58, RFC 2580, April 1999.

[RFC3411] Harrington, D., Presuhn, R., and B. Wijnen, "An Architecture for Describing Simple Network Management Protocol (SNMP) Management Frameworks", STD 62, RFC 3411, December 2002 .

[RFC3412] Case, J., Harrington, D., Presuhn, R., and B. Wijnen, "Message Processing and Dispatching for the Simple Network Management Protocol (SNMP) ", STD 62, RFC 3412, December 2002 . 
[RFC3413] Levi, D., Meyer, P., and B. Stewart, "Simple Network Management Protocol (SNMP) Applications", STD 62, RFC 3413, December 2002.

[RFC3414] Blumenthal, U. and B. Wijnen, "User-based Security Model (USM) for version 3 of the Simple Network Management Protocol (SNMPV3)", STD 62, RFC 3414, December 2002.

[RFC5590] Harrington, D. and J. Schoenwaelder, "Transport Subsystem for the Simple Network Management Protocol (SNMP)", RFC 5590, June 2009.

\subsection{Informative References}

[RFC3410] Case, J., Mundy, R., Partain, D., and B. Stewart, "Introduction and Applicability Statements for InternetStandard Management Framework", RFC 3410, December 2002.

[RFC3415] Wijnen, B., Presuhn, R., and K. McCloghrie, "View-based Access Control Model (VACM) for the Simple Network Management Protocol (SNMP)", STD 62, RFC 3415, December 2002 .

[RFC3418] Presuhn, R., "Management Information Base (MIB) for the Simple Network Management Protocol (SNMP)", STD 62, RFC 3418, December 2002 .

[RFC3584] Frye, R., Levi, D., Routhier, S., and B. Wijnen, "Coexistence between Version 1, Version 2, and Version 3 of the Internet-standard Network Management Framework", $\mathrm{BCP} 74, \mathrm{RFC} 3584$, August 2003.

[RFC5592] Harrington, D., Salowey, J., and W. Hardaker, "Secure Shell Transport Model for the Simple Network Management Protocol (SNMP)", RFC 5592, June 2009. 
Appendix A. Notification Tables Configuration

The SNMP-TARGET-MIB and SNMP-NOTIFICATION-MIB [RFC3413] are used to configure notification originators with the destinations to which notifications should be sent.

Most of the configuration is Security-Model-independent and Transport-Model-independent.

The values we will use in the examples for the five model-independent security and transport parameters are:

transportDomain $=$ snmpSSHDomain

transportAddress $=192.0 .2 .1: 5162$

securityModel $=$ Transport Security Model

securityName = alice

securityLevel = authPriv

The following example will configure the notification originator to send informs to a notification receiver at 192.0.2.1:5162 using the securityName "alice". "alice" is the name for the recipient from the standpoint of the notification originator and is used for processing access controls before sending a notification.

The columns marked with an "*" are the items that are Security-Modelspecific or Transport-Model-specific.

The configuration for the "alice" settings in the SNMP-VIEW-BASEDACM-MIB objects are not shown here for brevity. First, we configure which type of notification will be sent for this taglist (toCRTag).

In this example, we choose to send an Inform. snmpNotifyTable row: snmpNotifyName CRNotif snmpNotifyTag toCRTag snmpNotifyType inform snmpNotifystorageType nonVolatile snmpNotifyColumnstatus createAndGo

Then we configure a transport address to which notifications associated with this taglist will be sent, and we specify which snmpTargetParamsEntry will be used (toCR) when sending to this transport address. 


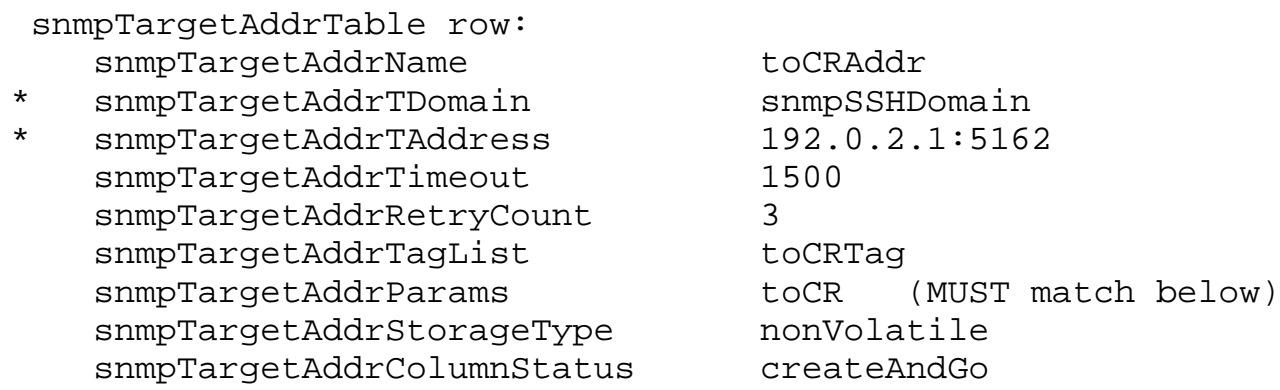

Then we configure which principal at the host will receive the notifications associated with this taglist. Here, we choose "alice", who uses the Transport Security Model. snmpTargetParamsTable row: snmpTargetParamsName toCR snmpTargetParamsMPModel SNMPv3

* snmpTargetParamsSecurityModel TransportSecurityModel snmpTargetParams SecurityName "alice" snmpTargetParams SecurityLevel authPriv snmpTargetParamstorageType nonVolatile snmpTargetParamsRowstatus createAndGo

A.1. Transport Security Model Processing for Notifications

The Transport Security Model is called using the generateRequestMsg() ASI, with the following parameters (those with an * are from the above tables):

\begin{tabular}{|c|c|c|}
\hline \multicolumn{2}{|c|}{$\begin{array}{l}\text { statusInformation }= \\
\text { generateRequestMsg( }\end{array}$} & -- success or errorIndication \\
\hline IN & messageProcessingModel & *snmpTargetParamsMPModel \\
\hline IN & globalData & message header, admin data \\
\hline IN & maxMessageSize & of the sending SNMP entity \\
\hline IN & transportDomain & *snmpTargetAddrTDomain \\
\hline IN & transportAddress & *snmpTargetAddrTAddress \\
\hline IN & securityModel & *snmpTargetParams SecurityModel \\
\hline IN & securityEngineID & immaterial; TSM will ignore. \\
\hline IN & securityName & snmpTargetParams SecurityName \\
\hline IN & securityLevel & -- *snmpTargetParams SecurityLevel \\
\hline IN & scopedPDU & -- message (plaintext) payload \\
\hline OUT & securityParameters & -- filled in by Security Module \\
\hline OUT & wholeMsg & complete generated message \\
\hline OUT & wholeMsgLength & length of generated message \\
\hline OUT & tmStateReference & -- reference to transport info \\
\hline
\end{tabular}


The Transport Security Model will determine the Transport Model based on the snmpTargetAddrTDomain. The selected Transport Model will select the appropriate transport connection using the tmStateReference cache created from the values of snmpTargetAddrTAddress, snmpTargetParamsSecurityName, and snmpTargetParams SecurityLevel.

Appendix B. Processing Differences between USM and Secure Transport

USM and secure transports differ in the processing order and responsibilities within the RFC 3411 architecture. While the steps are the same, they occur in a different order and might be done by different subsystems. The following lists illustrate the difference in the flow and the responsibility for different processing steps for incoming messages when using USM and when using a secure transport. (These lists are simplified for illustrative purposes, and do not represent all details of processing. Transport Models MUST provide the detailed elements of procedure.)

With USM, SNMPV1, and SNMPV2c Security Models, security processing starts when the Message Processing Model decodes portions of the ASN.1 message to extract header fields that are used to determine which Security Model will process the message to perform authentication, decryption, timeliness checking, integrity checking, and translation of parameters to model-independent parameters. By comparison, a secure transport performs those security functions on the message, before the ASN.1 is decoded.

Step 6 cannot occur until after decryption occurs. Steps 6 and beyond are the same for USM and a secure transport.

B.1. USM and the RFC 3411 Architecture

1) Decode the ASN.1 header (Message Processing Model).

2) Determine the SNMP Security Model and parameters (Message Processing Model).

3) Verify securityLevel (Security Model).

4) Translate parameters to model-independent parameters (Security Model).

5) Authenticate the principal, check message integrity and timeliness, and decrypt the message (Security Model). 
6) Determine the pduType in the decrypted portions (Message Processing Model).

7) Pass on the decrypted portions with model-independent parameters.

B.2. Transport Subsystem and the RFC 3411 Architecture

1) Authenticate the principal, check integrity and timeliness of the message, and decrypt the message (Transport Model).

2) Translate parameters to model-independent parameters (Transport Model).

3) Decode the ASN.1 header (Message Processing Model).

4) Determine the SNMP Security Model and parameters (Message Processing Model).

5) Verify securityLevel (Security Model).

6) Determine the pduType in the decrypted portions (Message Processing Model).

7) Pass on the decrypted portions with model-independent security parameters.

If a message is secured using a secure transport layer, then the Transport Model will provide the translation from the authenticated identity (e.g., an SSH user name) to a human-friendly identifier (tmSecurityName) in step 2. The Security Model will provide a mapping from that identifier to a model-independent securityName. 


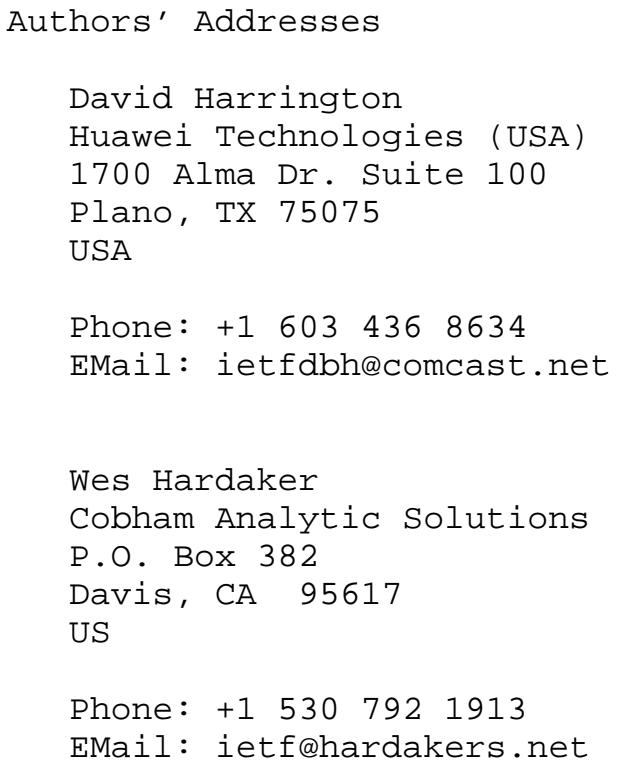

EMail: ietf@hardakers.net 